\title{
Subacute necrotizing encephalomyelopathy Its relationship to central pontine myelinolysis
}

\author{
AMIN A. FARIS' AND LORAN D. FLECKENSTEIN \\ From the Department of Pathology, Baptist Memorial Hospital, and the Department of Neurology, \\ The University of Tennessee College of Medicine, Memphis, Tennessee, U.S.A.
}

SUMMARY Clinical and neuropathological features of a case of subacute necrotizing encephalomyelopathy are discussed and compared with the features of central pontine myelinolysis. A hypothesis is offered relating the two diseases to a common aetiological factor.

Subacute necrotizing encephalomyelopathy (SNE) is a rare neurological disorder of unknown aetiology first reported by Leigh (1951). The purpose of this report is to present the clinical and neuropathological findings of an additional case of SNE and to explore a possible relationship between this entity and central pontine myelinolysis (CPM), a disorder of the central nervous system described by Adams, Victor, and Mancall (1959).

\section{CASE REPORT}

A.T. was the only child, a girl, born to healthy parents on 16 October 1959. The father was 37 years old and the mother was one year younger. The paternal and maternal family histories were non-contributory. No illness had been recorded by the mother during pregnancy. Prenatal care was adequate. Delivery was at full-term after three hours of spontaneous labour under local anaesthesia and without forceps. The birth weight was $7 \mathrm{lb} .6 \mathrm{oz} .(3 \cdot 3 \mathrm{~kg})$ and length was 21 in. $(52 \cdot 2 \mathrm{~cm})$. The child breathed spontaneously, cried, moved all extremities, had no jaundice, cyanosis, convulsions, paralysis, or stupor. The child had a good sucking reflex. She had a heart murmur which the local physician described as functional.

The child was bottle-fed from birth, was started on solid food at 3 weeks and began receiving supplementary vitamins at the age of 1 year. It was noted that the mother never successfully gave the child meat or eggs as part of her regular diet.

A sarvey of the development milestones revealed that the child was able to hold her head shortly after birth, able to turn over at 3 weeks, and able to crawl at 6 months. She stood at 7 months, was able to stand with support at 10 months, and walked without support at 18 months. Her weight increase was normal up to the age of 5 months, after which it dropped precipitously. The mental development of the child revealed that she

${ }^{1}$ Reprint requests to: Dr. Faris, 899 Madison Avenue, Memphis, Tennessee 38103, U.S.A. was speaking single words at the age of 2 years and complete sentences by the age of 3 years.

At about the age of 3 years, it was noted that her right eye turned outward and remained so for a period of 6 weeks. After this, it returned to normal position. In January 1963, she refused solid foods for a period of about one month. In May 1963, the child was admitted to a hospital for cardiac evaluation. She was found to have a mild degree of aortic stenosis. She was dismissed with no specific therapy. In July 1963, the parents noted a reduction in the degree of the child's ability to speak. For seven weeks before her second and last admission to the hospital on 3 October 1963, the child would take liquids only. She also became unable to walk without support. In addition, the parents noted a grunting type of respiration.

On admission to the hospital at the age of 3 years and 11 months, the child measured $33 \frac{3}{4}$ inches $(83.2 \mathrm{~cm})$ and weighed $181 \mathrm{lb}(8 \cdot 3 \mathrm{~kg})$. She appeared to be thin, welldeveloped, and was alternately apathetic and anxious. The mouth, throat, and skin appeared healthy. She had no palpable masses and there was no hepatosplenomegaly. The lungs were normal. There was a cardiac high-pitched ejection murmur in the pulmonary artery region. The pupils were equal, round, and reactive to light. The extraocular muscles functioned normally, although the child was noted to have mild nystagmus on lateral gaze. The optic discs had a sharp edge and appeared pale. The patient's gait was wide-based and unsteady. She could only walk with assistance. The deep tendon reflexes were normal. The plantar response was flexor on both sides. The child had a gross tremor that was exaggerated by excitement. She could speak only a few words clearly and was not able to express herself in sentences. Although the child demonstrated good muscle bulk and tone for the state of her nutrition, she was poorly coordinated.

On admission, the white blood count was $9,800 / \mathrm{c}$. mm with a normal differential, haemoglobin was $11.7 \mathrm{~g} / 100$ ml., haematocrit $41 \%$. Urinalysis was negative except for a positive reaction for ketone bodies. Blood urea nitrogen 
was $14 \mathrm{mg} / 100 \mathrm{ml}$., fasting blood sugar $111 \mathrm{mg} / 100 \mathrm{ml}$. Serum sodium was $138 \mathrm{~m}$-equiv/l., serum potassium $3.8 \mathrm{~m}$-equiv/l., chloride $102 \mathrm{~m}$-equiv/l., and $\mathrm{CO}_{2} 26 \mathrm{~m}$ equiv/l. Spinal puncture revealed a clear, colourless fluid under normal pressure, no cells, protein $18 \mathrm{mg}$, and sugar $99 \mathrm{mg} / 100 \mathrm{ml}$. Serum electrophoresis was normal. Electroencephalography revealed a moderately well-organized record that was generally slow with occasional spike waves occurring from either temporal region during sleep; a rare occipital spike was also described. The skull radiographs were normal. Chest films suggested pulmonary emphysema. The patient's bone age was 3 years.

The patient's short course in the hospital was characterized by excessive somnolence and withdrawal. Feeding was limited to liquids by mouth. On occasion, restlessness became outstanding and sedation with phenobarbitone was administered. On the fourth hospital day, she went into a period of respiratory embarrassment with ensuing cyanosis, necessitating mechanical respiratory assistance. A day later she was found dead.

NECROPSY EXAMINATION A necropsy was performed four hours after death. The lungs were atelectatic and microscopically demonstrated acute bronchial pneumonia. The heart showed moderate hypertrophy of the right ventricle. The aortic valve and aorta appeared normal.

Central nervous system The brain weighed $950 \mathrm{~g}$ in the fresh state. The cerebral hemispheres were symmetrical, the leptomeninges were transparent and delicate. The sulci and gyri were normal. The blood vessels of the base of the brain, the cranial nerves, and external appearance of the brain-stem and cerebellar hemisphere showed no unusual features. Sections of the brain disclosed small, pale brown areas of spongy degeneration symmetrically placed in the walls of the posterior portion of the third ventricle, measuring about $3 \mathrm{~mm}$ in width. The subthalamic nuclei were reduced to about one-half the normal size and had a grey colour. The substantia nigra had no pigment and was also about half its normal size on either side. Areas of grey discolouration that had a pitted appearance involved the periaqueductal grey matter. Similar lesions involved the tegmentum of the midbrain in the region of the third and fourth cranial nerve nuclei and reticular formation. The superior and inferior colliculi also demonstrated similar changes. Areas of spongy degeneration were also present in the subependymal region of the fourth ventricle in the pons and medulla extending for about 2 to $3 \mathrm{~mm}$. These morphological changes in the tegmentum of the pons also included the fifth nerve nucleus and the nuclei of the sixth and seventh cranial nerves as well as the reticular nuclei. In the medulla, in addition to the subependymal ventricular involvement, the tegmentum and medial portion of the inferior olivary nuclei showed changes as described. Sections of the cerebellum showed brownish discolouration of the roof nuclei. The lateral, third, and fourth ventricles were not enlarged. The mammillary bodies showed no changes. The external appearance and sections of the spinal cord were normal.

Microscopic examination confirmed the presence of lesions in the areas described. In addition, however, the red nucleus appeared involved. The lesions were characterized by rarefaction and loss of neurones that was partial or complete. In many areas persistence of normal appearing neurones intermixed with neurones suggesting chromatolytic changes were apparent. There was marked loss of myelin in the involved areas, but silver impregnation studies demonstrated minimal degrees of axonal loss. No neuroaxonal dystrophy or spheroid bodies were encountered. The tissues within the areas of degeneration consisted, for the most part, of a network of glial fibres where there was a moderate increase in the number and size of astrocytes. The microglial reaction was, in most areas, moderate and more prominently displayed in evolution toward phagocyte formation in the dentate and roof nuclei of the cerebellum. The blood vessels in all regions involved appeared to be increased in number. Many of them showed an increase in the cells of their walls and, in some, fibrous thickening was also noted.The lesions around the third ventricle demonstrated a few petechial haemorrhages, but no blood pigment was seen. The mammillary bodies were normal. The spinal cord demonstrated no unusual features.

\section{DISCUSSION}

Subacute necrotizing encephalomyelopathy was firs described by Leigh (1951). Since then 33 cases have been described and recently reviewed and analysed by Lakke, Ebels, and Ten Thye (1967). Clinically the disease is characterized by psychomotor retardation, ataxia, ophthalmoplegia, muscular weakness, and incoordination, resulting in death due to respiratory embarrassment. The duration of the illness varied from an acute to a subacute course but few cases in the juvenile age group ran a chronic course. The neuropathological picture is characteristically that of bilaterally symmetrical spongiform degeneration of the brain-stem nuclei, more intensely noted in the periventricular regions, cerebellar roof nuclei and, characteristically, sparing of the mammillary bodies. Microscopically the lesions are characterized by periaxial demyelination, with preservation of the axons, relative preservation of the neurones in the involved areas with varying degrees of glial and microglial cell proliferation, and prominent vascularization. These features led Feigin and Wolf (1954) to publish three cases in 1954 under the heading of 'A Disease of Infants Resembling Chronic Wernicke's Encephalopathy', a concept that is a focal point of interrelationship with what perhaps are related disorders, as will be seen later.

The aetiology of subacute necrotizing encephalomyelopathy remains unknown. From their observations, Feigin and Wolf (1954) suggested an inherent enzymatic metabolic disorder blocking the utilization of thiamine. Two of their cases were siblings, which lends support to the idea of an inherited fault 
in metabolism. Familial incidence of the disease has also been reported by Namiki (1965) and Richter (1957). More recently, Greenhouse and Schneck (1968), after studying one case for metabolic defects related to thiamine deficiency and reviewing the literature of reported cases where lactic acid and pyruvic acid were found elevated, concluded that at the present time evidence for thiamine deficiency or lack of thiamine utilization in carbohydrate metabolism cannot be implicated as a cause of the disease. The authors suggest the need for detailed examination of the carbohydrate pathways of metabolism in an extensive manner in the hope of possibly realizing the causative metabolic factor. Dietary deficiency, although suggested as a possible cause from the history of our patient, would be suggested as a result rather than the cause of the disorder by the cases in the literature (Lakke et al., 1967).

From the available data, it may be speculated that this disorder is due to a metabolic defect preferentially causing periaxial demyelination in selectively vulnerable areas in the central nervous system, perhaps due to selective metabolic fault in the oligodendrogliocyte.

Adams et al., (1959) described central pontine myelinolysis in four adult cases, three of whom were alcoholic and one who had systemic neoplasia. Since then, about 60 cases have been reported in the literature. The disease runs an acute and subacute course characterized by bulbar and long-tract signs, coma and death. Pathologically, the entity is characterized by varying sized triangular lesions in the basis pontis that histologically show periaxial demyelination, relative preservation of axons, preservation of neurones within the affected areas, macrophage activity, and mild glial proliferation, features very similar to those described for subacute necrotizing encephalomyelopathy, lacking, however, the vascular proliferation and most often only restricted to the pons, although some cases have been described to show involvement in the basal ganglia and cerebellum (Mathieson and Olszewski. 1960). The aetiological factors leading to central pontine myelinolysis are yet to be clarified. Adams et al. suggested alcohol as a possible agent and, in addition, suggested the relationship of the disease to Wernicke's encephalopathy. However, a history of alcoholism is not available in about onehalf of the cases reported (Kepes, Reece, and Oxley, 1965), the remainder having a variety of systemic disorders, such as carcinoma, tuberculosis, and collagenosis. As Aleu and Terry (1963) pointed out, alcoholism is very common and of long standing among humans, and only recently has CPM been discovered, suggesting an added, if not independent, factor, probably external such as the variety of iatrogenic agents used in maintaining chronically ill patients. The case of Kepes et al. (1965) is of great interest as is case 2 of Matsuoka (1966), both of which followed operations on craniopharyngioma in childhood where alcoholism was not present; rather, severe electrolyte imbalance followed surgery, from which the authors were led to believe that metabolic disorders leading toward electrolyte imbalance may lead to preferential involvement of the oligodendroglia in the central pons, leading to myelin sheath disintegration. This hypothesis was also favoured by Chason, Landers, and Gonzalez (1964), Behar, Bental, and Aviram (1964) and Berry and Olszewski (1963). The question of localization of the lesion to the central pons becomes an important point about which many authors have speculated. Mathieson and Olszewski (1960) suggested the concept of 'pathoclisis' or selective vulnerability as forwarded by the Vogts, a point of view that we believe is theoretically quite tenable. The possible primary vascular involvement has been dismissed by Adams et al. and several other workers because of the unclassical picture of the disease, in particular the preservation of neurones and lack of evidence of ischaemic changes. The case of Rosman, Kakulas, and Richardson (1966), a 7-year-old boy who had acute leukaemia, was treated with cellular toxins, cortical steroids, and antibiotics. The authors point out the importance of the possible role of antibiotics and other medications used in the treatment of such cases leading to a localized metabolic deficiency in the central pons and, in addition, the possible contribution of the neoplastic state contributing to further metabolic aberrations. The three cases of Schneck (1966) in instances of renal transplantation, in addition to the classical features of CPM, demonstrated neuroaxonal dystrophy of the spheroid type seen in human deficiency states leading Schneck to believe that the disease indeed had a metabolic background, possibly a deficiency state relating to a localized portion of the central nervous system. Shiraki and Oda (1968) have described cases of CPM in hepatocellular disorders. The third case of Matsouka (1966) had Wilson's disease. This further indicated to the authors the role of metabolic alterations that may be responsible for the resultant neuropathological changes.

The fact remains, however, that all of the disorders mentioned in association with central pontine myelinolysis have long been known and studied at necropsy without discovery of the disease process. This fact would indicate that recent additional factors have been introduced to precipitate a specific histopathological central nervous system change not previously encountered. The same argument may be offered for the disorder of Leigh's subacute necro- 
tizing encephalomyelopathy, which, in scope of anatomical involvement, is wider than central pontine myelinolysis, but perhaps only as part of a spectrum of the same underlying process. The clinical picture and neuropathological changes are quite similar and have suggested to a number of authors a link with Wernicke's encephalopathy, so that a mutual bond of aetiological factors, when better studied and understood, might lead to the realization of some of the aspects leading to periaxial demyelination, a feature also prominent in multiple sclerosis.

The authors wish to express their gratitude to Dr. John Kepes of the Department of Pathology, University of Kansas, for making the pathological material accessible.

\section{REFERENCES}

Adams, R. D., Victor, M., and Mancall, E. L. (1959). Central pontine myelinolysis. A hitherto undescribed disease occurring in alcoholic and malnourished patients. Arch. Neurol. Psychiat. (Chic.), 81, 154-172.

Aleu, F. P., and Terry, R. D. (1963). Central pontine myelinolysis. Arch. Path., 76, 140-146.

Behar, A., Bental, E., and Aviram, A. (1964). Central pontine myelinolysis. Acta neuropath. (Berl.), 3, 343-350.

Berry, K., and Olszewski, J. (1963). Central pontine myelinolysis. Neurology (Minneap.), 13, 531-537.

Chason, J. L., Landers, J. W., and Gonzalez, J. E. (1964). Central pontine myelinolysis. J. Neurol. Neurosurg. Psychiat., 27, 317-325.
Feigin, I., and Wolf, A. (1954). A disease in infants resembling chronic Wernicke's encephalopathy. J. Pediat., 45, 243-263.

Greenhouse, A. H., and Schneck, S. A. (1968). Subacute necrotizing encephalomyelopathy: $A$ reappraisal of the thiamine deficiency hypothesis. Neurology (Minneap.), 18, 1-8.

Kepes, J. J., Reece, C. A., and Oxley, D. K. (1965). Central pontine myelinolysis in a 7-year-old boy. J. Neurol. Neurosurg. Psychiat., 28, 39-47.

Lakke, J. P. W. F., Ebels, E. J., and Ten Thye, O. J. (1967). Infantile necrotizing encephalomyelopathy (Leigh). Arch. Neurol. (Chic.), 16, 227-231.

Leigh, D. (1951). Subacute necrotizing encephalomyelopathy in an infant. J. Neurol. Neurosurg. Psychiat., 14, 216-221.

Mathieson, G., and Olszewski, J. (1960). Central pontine myelinolysis with other cerebral changes. Neurology (Minneap.), 10, 345-354.

Matsuoka, T. (1966). Central pontine myelinolysis (abstract). Clin. Neurol., 6, 101.

Namiki, H. (1965). Subacute necrotizing encephalomyelopathy. Arch. Neurol. (Chic.), 12, 98-107.

Richter, R. B. (1957). Infantile subacute necrotizing encephalopathy with predilection for the brain stem. $J$. Neuropath. exp. Neurol., 16, 281-307.

Rosman, N. P., Kakulas, B. A., and Richardson, E. P., Jr. (1966). Central pontine myelinolysis in a child with leukemia. Arch. Neurol. (Chic.), 14, 273-280.

Schneck, S. A. (1966). Neuropathological features of humañ organ transplantation. J. Neuropath. exp. Neurol., 258 18-39.

Shiraki, H., and Oda, M. (1968). Neuropathology of hepato $\stackrel{9}{~}$ 을 cerebral disease with emphasis upon comparative studies. In: Pathology of the Nervous System, p. 10890 Vol. 1. Editor, J. Minckler. McGraw-Hill: New York 